by solicitors contemplating a CFA. In these cases, accurate risk assessment is essential to the solicitor, particularly because after-the-event insurance is currently available for only a very specific category of personal injury cases. At this stage, insurers are unclear about how they can expand the existing system to cover other types of case, meaning that solicitors who choose to accept those cases must run them bare of insurance.

Given the problems potential litigants now face with the court system, alternative dispute resolution (ADR) methods are increasing in popularity. ADR offers several alternatives to traditional court methods and a number of opportunities for expert involvement. Within the scope of $\mathrm{ADR}$. experts can act as advisors or offer expert appraisal of a technical issue. Parties may also agree to an 'expert determination', where an expert rules conclusively on the issue for them. or ask the experts to take on the role of mediator.

Experts interested in obtaining further details can contact the Society of Expert Witnesses on (0345) 023014 or write to PO Box 345, Newmarket CB8 7TU.

VANessa Lumpkin, Professional Liaison, Society of Expert Witnesses, PO Box 345, Newmarket CB8 7TU

\section{Health of the Nation Outcome Scales for People with Learning Disabilities}

Sir: Further to the publication of the original paper 'HoNOS in long-stay patients with learning disabilities' (Psychiatric Bulletin, May 1988, 22. 306-308), we are writing to keep you informed of recent developments with regard to the piloting of the Health of the Nation Outcome Scales for People with Learning Disabilities (HoNOS-LD).

HoNOS-LD is the result of a collaboration between the Department of Health, the Royal College of Psychiatrists and the Centre for Outcomes, Research and Clinical Effectiveness (CORE) at the British Psychological Society, University College London. Like the generic HoNOS, HoNOS-LD is a set of scales designed to measure outcomes in a population of people with mental health needs. It retains the five-point scale of severity of problems, but has greater context reliability with regard to the associated needs of the client group. HoNOS-LD has 18 items, is designed for people irrespective of their degree of learning disability and should be used by trained professionals.

North Warwickshire National Health Service (NHS) Trust has allocated resources for the post of Regional Clinical Audit Coordinator who has responsibility for training, data analysis and dissemination in an area north of a line from the Wash to the Bristol Channel, while CORE have employed a researcher to take responsibility for the same in participating trusts in the south.

Following a training day, agreement was reached that HoNOS-LD would need to be tested rigorously for interrater reliability and sensitivity to change. Version 2 is currently being piloted at over 20 sites (all NHS trusts) throughout the United Kingdom.

It is expected that data analysis will have been completed in Autumn this year and, if the instrument is demonstrated to have acceptable reliability, will be made available by the Department of Health for use in routine clinical practice for people with learning disabilities and mental health needs.

DAvid MarTIN, Regional Clinical Audit Coordinator, and AsHOK RoY, Consultant Psychiatrist, HoNOS-LD, Brooklands, Coleshill Road, Marston Green, Birmingham B37 7HL

\section{The practice of evidence-based journal clubs}

Sir: Geddes (Psychiatric Bulletin, June 1998, 22. 337-338) states that an evidence-based journal club (EBJC) presentation can be prepared in two hours. This may be the case at the centre for evidence-based mental health in Oxford, but here in Britain's most northerly department of academic psychiatry we struggle to believe it. Perhaps our remoteness from such a centre of excellence slows our thinking, but we do not see how the process can be effected in such a brief time.

In Aberdeen we recently introduced an EBJC linked to the weekly case conference and we encourage junior staff to present. We find the preparation process takes considerably longer than Geddes allows, for several reasons. Many presenters lack experience in critical appraisal and require individual mentorship, not always available through their weekly educational supervision. The authors act as mentors, taking particular responsibility for helping trainees develop the required skills.

The clinical question is set three weeks ahead of the session by the consultant presenting the case and it is helpful for a mentor to be involved. The presenter requires guidance in the process of searching the literature databases and deciding which papers to select. Obtaining papers not held locally may take some weeks. Critical review may involve the trainee having to ask others for assistance in assessment of study methods, particularly statistics. Finally, the presenter needs to prepare materials and may wish to rehearse their presentation. 
Our EBJC is evolving into a successful academic forum for training and continuing professional development. The aims are to teach the process of identifying and assessing relevant evidence and to reach a clinical bottom line. We are concerned that even with a little practice two hours will not do justice to either aim.

Nicholas P. WALKer, Senior Registrar in General Psychiatry, Honorary Clinical Lecturer. PAUline MCCONVILle, Clinical Lecturer in Mental Health, Honorary Specialist Registrar, and IAN G. DEWAR, Senior Registrar in General Psychiatry. Honorary Clinical Lecturer. University of Aberdeen, Department of Mental Health. Clinical Research Centre, Royal Cornhill Hospital, Cornhill Road, Aberdeen, Scotland AB25 2ZH

\section{Medical staffing crisis in psychiatry}

Sir: I agree with Jenkins \& Scott's (Psychiatric Bulletin. April 1998, 22, 239-241) statement that the current crisis in medical staffing is a cause of serious concern. However, in their discussion of the possible reasons for this crisis they did not mention the increasing difficulties junior psychiatrists are currently facing in comparison with colleagues in other specialities. It is possible that psychiatry will become increasingly unpopular in the next decade because other competing specialities have made greater steps in rationalising their postgraduate training and career structure.

The introduction of the Calman reforms and the specialist registrar grade will significantly shorten and rationalise training in medicine, surgery and other hospital specialities. Conversely. these reforms have had a deleterious effect on psychiatry by lengthening the time spent in the poorly paid and perceived senior house officer grade.

Other specialities have made advances in lightening the burden of junior on-call duties. Innovations include, split shifts and a mandatory day off after being on-call. Unfortunately the burden of the junior psychiatrist continues to escalate, difficulties include: chronic bed shortages, violent patients, unhelpful casualty departments and the significant isolation of being on-call without a 'team' of peers.

We must be aware that factors such as career structure, time spent in junior grades and burden of on-call duties are important in the decision to choose a particular speciality. Such factors have been favourable in psychiatry, but recently have become less so. Unless this is acknowledged and rectified we will continue to see a decline in juniors entering our profession.

RAJEN SHAH, Specialist Registrar in Old Age Psychiatry, Dove Unit, Crawley Hospital, West Green Drive, West Sussex

\section{The Internet and the future of psychiatry}

Sir: Huang \& Alesi (1996) discuss the implications of the World Wide Web for both psychiatrists and their patients and easy access to information has been particularly emphasised. although the quality of information and effects on patients of accessing such data has been questioned (Suresh \& Lynch, 1998).

We wish to report a case that further highlights the potential dangers of how some information available on the Internet is being used. To our knowledge this is the first reported case in which a patient chose unusual suicide methods directly from a Web site on the Internet.

We treated a female patient who presented on two occasions to medical and psychiatric services having ingested roots of wolfsbane (Aconitum napellus), the most poisonous plant in the United Kingdom (Poisons Bureau Edinburgh). Among reported effects are bradycardia, hypotension, arrhythmias and death. On both occasions the patient required admission to and treatment on a coronary care unit for ventricular ectopics. On another occasion, once again accessed on the Internet, she injected approximately $2 \mathrm{ml}$ of lighter fuel into her left forearm. This resulted in severe, local cellulitis with massive swelling which compromised distal circulation necessitating surgical compartment relief and subsequent skin grafting. She maintained that both these methods were found on the Internet and recommended as being 'certain' and relatively quick.

While the patient was reluctant to reveal the exact Web site where she found these methods, a quick Yahoo search under the keyword 'suicide' produced a number of sites containing the relevant information. For example, the 'Doyerself Web site (http://www.inergy.com/doyerself) and the 'suicide methods' Web site (http://www. montana.com/personal/kujawa/rob/suicide_m ethods.html) contain information on both methods used and offer links to other 'relevant' sites. The suicide methods site alone provides detailed information on close to 100 different ways to commit suicide ranging from self-poisoning to taking an acid bath.

These are just a small example of a number of very alarming and inapproprlate sites, freely accessible to anyone. Psychiatrists need to be aware of the possibility of their patients. some of 